\title{
Predictors of First Dose of Immunoglobulin Resistance in Children with Kawasaki Disease
}

\author{
${ }^{1} X i$ 'an medical University, Xi'an 710021 \\ ${ }^{2}$ Children's Hospital of Shaanxi Provincial People's Hospital, Xi'an 710068 \\ ${ }^{3}$ School of Public Health, School of Medicine, Xi'an Jiaotong University, Xi'an 710061, China \\ ${ }^{4}$ Tamale Teaching Hospital, Tamale, Ghana \\ ${ }^{5}$ School of Medicine and Health Sciences, University for Development Studies, Tamale, Ghana \\ ${ }^{6}$ Shenyang Medical College, Shenyang, China \\ ${ }^{7}$ Feng Tian Hospital affiliated to Shenyang Medical College, Shenyang, China
}

Ma Lei ${ }^{1}$, Jiao Fu-Yong' ${ }^{2}$, Wang Jie-Min² ${ }^{2}$ Lin Jing ${ }^{3}$, Yan Xian-Peng ${ }^{2}$ and Alhaji Adam Abubakari ${ }^{4,5,6,7 *}$

Received Date: October 17, 2018; Published Date: November 29, 2018

*Corresponding author: Alhaji Adam Abubakari, Tamale Teaching Hospital, Tamale, Ghana, West Africa

\section{Abstract}

Objective: To explore the predictive factors of the first dose of IVIG resistance in children with Kawasaki disease.

Methods: The clinical data of 301 children with KD were retrospectively analyzed. They were divided into IVIG insensitive group and IVIG sensitive group. The basic data and laboratory indexes of the two groups were compared. The risk factors of IVIG resistance were determined by binary logistic regression analysis and receiver operating characteristic curves (ROC curves) were constructed to determine the optimal predictive threshold value of the risk factors and the corresponding sensitivity and specificity.

Results: Neutrophil ratio (N\%), basophil ratio (B\%), alanine aminotransferase (ALT), aspartate aminotransferase (AST), Procalcitonin (PCT) levels are higher in the IVIG insensitive group compared with the IVIG sensitive group $(\mathrm{P}<0.05)$ and lymphocyte ratio (L\%), monocyte ratio (M\%), hematocrit (HCT), mean erythrocyte hemoglobin amount ( $\mathrm{MCH})$, creatine kinase (CK), sodium concentration (Na+) levels are lower $(\mathrm{P}<0.05)$. Binary logistic regression analysis shows that AST and PCT are independent risk factors for IVIG resistance. AST $\geq 29.5 \mathrm{U} / \mathrm{L}$ holds the highest sensitivity and specificity for predicting IVIG resistance, which were $91.7 \%$ and $64.4 \%$, respectively. PCT $\geq 1.70 \mathrm{ng} / \mathrm{ml}$ predicts the highest efficacy of IVIG resistance, and the corresponding sensitivity and specificity are $91.7 \%$ and $62.3 \%$, respectively.

Conclusion: AST and PCT can be used as predictors of IVIG resistance in KD.

Keywords: Kawasaki Disease; Immunoglobulin; Resistance; Predictors

\section{Background}

Kawasaki disease (KD) is the most common cause of acquired heart disease in children. It is an acute, self-limiting vasculitis that mainly affects the small and medium arteries of the whole body. Long term consequences of undiagnosed and untreated KD in childhood would likely impact health care delivery systems in developing countries [1]. The pathogenesis of the disease has not yet been fully understood. We currently consider that it is the interaction between infectious factors and genetic susceptibility causing abnormal immune responses in the body that results in KD [2]. Intravenous immunoglobulin (IVIG) combined with aspirin is now recognized as the first-line standard therapy for Kawasaki disease and IVIG can reduce the incidence of coronary artery damages. However, there are still $10 \%-20 \%$ of children with Kawasaki disease do not respond to the first dose of IVIG [3], and it has been confirmed that they are associated with a higher risk of coronary artery diseases. In addition, medical and family burdens for children who have the first dose of IVIG resistance in KD are heavier. Therefore, it is particularly important to find factors that can predict the IVIG resistance of Kawasaki disease.
At present, there are many studies on the predictive factors of IVIG resistance in Kawasaki disease. Laboratory indicators which have been found for predicting IVIG non-responsive KD include C-reactive protein (CRP), erythrocyte sedimentation rate (ESR), hemoglobin concentration (HGB), platelet count(PLT), white blood cell count (WBC), N\%, percentage of polymorphonuclear leukocytes (PMN\%), percentage of segmented neutrophils (SEG\%), neutrophil-lymphocyte ratio (NLR), ALT, AST, albumin (ALB), total bilirubin (TBil), lactate dehydrogenase(LDH), N-terminal brain natriuretic peptide precursor (NT-proBNP), Na+,interleukin6(IL-6),interleukin-10 (IL-10), etc. But because of differences in genetic background, geographical environment, epidemiology such as infection, and the lack of relevant single-center/multicenter retrospective study in this region; in addition, relevant studies have more or less limitations such as single inclusion, inability to be detected routinely, and poor specificity. Therefore, this study conducted a more comprehensive statistical analysis of clinical features and laboratory routine testing indicators which 


\section{Novel Approaches in Drug Designing \& Development}

may be related to IVIG resistance in order to find more valuable predictors.

\section{Methods}

\section{Research Object}

A total of 301 children with KD diagnosed and hospitalized in the Children's Hospital of Shaanxi Provincial People's Hospital from May 2014 to May 2018 were retrospectively analyzed in this study. All subjects were treated with a large dose of IVIG $(2 \mathrm{~g} / \mathrm{kg}$, infused within 12 hours) and oral aspirin $(30-50 \mathrm{mg} / \mathrm{kg} \cdot \mathrm{d})$ after diagnosis. The aspirin dose was reduced to $3-5 \mathrm{mg} / \mathrm{kg} \cdot \mathrm{d}$ after the body temperature was normal for $36 \mathrm{~h}$.

\section{Inclusion and Exclusion Criteria for Selected Subjects}

Inclusion Criteria: The diagnosis of KD was based on the 2004 Kawasaki disease diagnosis guide issued by the American Heart Association [4], that is, fever $\geq 5$ days and 5 major clinical manifestations $\geq 4$, or major clinical manifestations $<4$ but echocardiography or cardiovascular angiography shows coronary artery dilatation or coronary artery aneurysm, and KD can be diagnosed after excluding other diseases with similar manifestations.

\section{Exclusion criteria}

a. Children who could not be diagnosed as KD

b. Children who did not apply IVIG and were not clearly classified as IVIG sensitive group or IVIG insensitive group

c. Children with recurrent KD

d. Children with KD who were re-admitted after the first hospitalization was ineffective

e. Children with KD complicated with other vascular inflammatory diseases.

\section{Grouping Criteria}

All subjects were divided into IVIG insensitive group and IVIG sensitive group according to the presence or absence of IVIG resistance. The diagnostic criteria for IVIG resistance was based on the recommendations of the 2007 National KD Symposium in China, that is, apart from secondary infections, non-regression of fever 36 hours after IVIG treatment (body temperature $\geq 38^{\circ} \mathrm{C}$ ) or recurrence of symptoms 2-7 days after administration (fever with at least one major KD symptom) could be diagnosed as IVIG unresponsive $\mathrm{KD}$.

\section{Clinical Data Collection Content}

Basic Information: Including age, sex, weight, place of usual residence(town/country), maximum body temperature during fever(Tmax), time from onset to use of IVIG(time).

Laboratory Indicators: All were obtained when the first examination before IVIG treatment and at admission for children patients.

Blood routine: $\mathrm{WBC}, \mathrm{N} \%, \mathrm{~L} \%, \mathrm{M} \%$, eosinophil ratio(E\%), $\mathrm{B} \%$, HGB, HCT, mean erythrocyte volume(MCV), $\mathrm{MCH}$, mean erythrocyte hemoglobin concentration(MCHC), PLT; Biochemistry: ALT, AST, CK, creatine kinase MB isoenzyme (CK-MB), LDH, alpha hydroxybutyrate dehydrogenase(HBDH),potassium ion concentration $(\mathrm{K}+), \mathrm{Na}+$.

Inflammatory Index: CRP, PCT, ESR. The study was reviewed and approved by the hospital ethics committee and the informed consent of the parents of the children was obtained.

\section{Statistical Analysis}

SPSS 19.0 software was used for statistical analysis. Handling of missing data: replace missing data with a linear trend method at the point. The count data were expressed as the number of cases and the comparison between groups was performed byx 2 test; measurement data with normal distribution were expressed as mean \pm standard deviation ( $x \pm s$ ) and two independent samples $t$ test was used for comparison between groups; measurement data with abnormal distribution were expressed as median(interquartile range) and compared by using rank sum test. All laboratory indicators included were subjected to binary logistic regression analysis to determine the independent risk factors associated with IVIG resistance. The receiver operating characteristic curve (ROC curve) was used to determine the optimal predictive threshold and sensitivity and specificity of risk factors. $\mathrm{P}<0.05$ is considered statistically significant.

\section{Research Result}

A total of 301 children with KD (191 males, 110 females) were included in the study, including 12 patients in the IVIG insensitive group and 289 patients in the IVIG sensitive group. The median age was 24 months (age range 12-36 months).

\section{Comparison of Basic Data and Laboratory Indicators Between IVIG Insensitive Group And Sensitive Group}

Table 1: Comparison of basic information and laboratory indicators between IVIG insensitive and sensitive group

\begin{tabular}{|c|c|c|c|c|}
\hline & $\begin{array}{c}\text { IVIG insensitive group } \\
(\mathbf{n = 1 2})\end{array}$ & $\begin{array}{c}\text { IVIG sensitive group } \\
(\mathbf{n = 2 8 9})\end{array}$ & $\chi^{2} / \mathbf{t} / \mathbf{Z}$ value & P value \\
\hline Sex (male / female) & $8 / 4$ & $183 / 106$ & 0 & $>0.05$ \\
\hline Place of usual residence (town/rural) & $6 / 6$ & $160 / 129$ & 0.134 & 0.714 \\
\hline Age (months) & $18(12-45)$ & $24(12-36)$ & -0.127 & 0.899 \\
\hline Weight(Kg) & $13(11-16.75)$ & $12.5(10-16)$ & -0.521 & 0.603 \\
\hline $\left.\mathrm{T}_{\max }{ }^{0} \mathrm{C}\right)$ & $39.9(39.43-40)$ & $39.5(39-39.95)$ & -1.512 & 0.131 \\
\hline time(d) & $7(6-7.65)$ & $7(7-9)$ & -1.677 & 0.094 \\
\hline
\end{tabular}


Novel Approaches in Drug Designing \& Development

\begin{tabular}{|c|c|c|c|c|}
\hline $\mathrm{WBC}\left(\times 10^{9} / \mathrm{L}\right)$ & $12.57(10.14-16.15)$ & $13.20(10.21-17.60)$ & -0.538 & 0.59 \\
\hline$N(\%)$ & $72.00 \pm 18.50$ & $60.83 \pm 16.40$ & -2.299 & 0.022 \\
\hline $\mathrm{L}(\%)$ & $20.50 \pm 13.55$ & $28.94 \pm 13.91$ & 2.061 & 0.04 \\
\hline $\mathrm{M}(\%)$ & $4.50(2.00-8.50)$ & $7.80(5.00-10.00)$ & -2.040 & 0.041 \\
\hline $\mathrm{E}(\%)$ & $1.00(0.00-1.76)$ & $2.00(0.00-3.00)$ & -1.814 & 0.07 \\
\hline $\mathrm{B}(\%)$ & $0.08(0.00-0.79)$ & $0.00(0.00-0.00)$ & -2.788 & 0.005 \\
\hline $\operatorname{RBC}\left(\times 10^{12} / \mathrm{L}\right)$ & $4.06 \pm 0.33$ & $4.11 \pm 0.42$ & 0.430 & 0.668 \\
\hline $\operatorname{HGB}(\mathrm{g} / \mathrm{L})$ & $103.33 \pm 12.12$ & $109.33 \pm 11.36$ & 1.788 & 0.075 \\
\hline НCT(\%) & $0.33(0.30-0.35)$ & $0.34(0.32-0.36)$ & -2.390 & 0.017 \\
\hline MCV(fL) & $81.34 \pm 5.66$ & $84.17 \pm 4.93$ & 1.939 & 0.053 \\
\hline $\mathrm{MCH}(\mathrm{pg})$ & $26.35(24.43-26.66)$ & $26.70(25.75-27.70)$ & -2.082 & 0.037 \\
\hline $\operatorname{MCHC}(\mathrm{g} / \mathrm{L})$ & $314.01(311.25-320.75)$ & $317.00(308.50-325.50)$ & -0.416 & 0.677 \\
\hline $\operatorname{PLT}\left(\times 10^{9} / \mathrm{L}\right)$ & $395.83 \pm 259.55$ & $366.86 \pm 131.03$ & -0.385 & 0.708 \\
\hline $\operatorname{ALT}(\mathrm{U} / \mathrm{L})$ & $68.50(25.00-200.00)$ & $20.00(12.00-38.83)$ & -3.248 & 0.001 \\
\hline AST(U/L) & $42.20(31.50-61.50)$ & $26.00(20.00-34.00)$ & -3.399 & 0.001 \\
\hline $\mathrm{CK}(\mathrm{U} / \mathrm{L})$ & $23.50(19.00-28.75)$ & $41.00(24.00-65.44)$ & -3.084 & 0.002 \\
\hline CK-MB(U/L) & $18.00(14.50-21.50)$ & $20.00(16.00-25.00)$ & -1.086 & 0.277 \\
\hline LDH(U/L) & $265.00(231.00-309.75)$ & $266.00(227.00-297.50)$ & -0.430 & 0.667 \\
\hline $\mathrm{HBDH}(\mathrm{U} / \mathrm{L})$ & $184.29(164.25-232.00)$ & $195.00(167.00-226.50)$ & -0.513 & 0.608 \\
\hline $\mathrm{K}^{+}(\mathrm{mmol} / \mathrm{L})$ & $4.68(4.03-5.31)$ & $4.79(4.50-5.10)$ & -1.385 & 0.166 \\
\hline $\mathrm{Na}^{+}(\mathrm{mmol} / \mathrm{L})$ & $134.82(131.50-136.66)$ & $137.00(135.73-138.00)$ & -2.699 & 0.007 \\
\hline $\mathrm{CRP}(\mathrm{mg} / \mathrm{L})$ & $62.30(42.35-123.03)$ & $61.12(29.66-85.55)$ & -0.735 & 0.463 \\
\hline PCT(ng/ml) & $2.64(1.72-14.56)$ & $0.60(0.21-1.75)$ & -3.363 & 0.001 \\
\hline $\operatorname{ESR}(\mathrm{mm} / \mathrm{h})$ & $76.00 \pm 36.02$ & $59.55 \pm 29.30$ & -1.888 & 0.06 \\
\hline
\end{tabular}

Note: Tmax: Highest Body Temperature During Fever; Time: Time from Onset to Use of IVIG; WBC: White Blood Cell Count; N:Neutrophil Ratio; L:Lymphocyte Ratio; M: Monocyte Ratio; E:Eosinophil Ratio; B:Basophil Ratio; RBC: Red Blood Cell Count; HGB: Hemoglobin Concentration; HCT: Hematocrit; MCV: Mean Erythrocyte Volume; $\mathrm{MCH}$ : Mean Erythrocyte Hemoglobin Amount; MCHC: Mean Erythrocyte Hemoglobin Concentration; PLT: Platelet Count; ALT: Alanine Aminotransferase; AST: Aspartate Aminotransferase; CK: Creatine Kinase; CK-MB: Creatine Kinase MB isoenzyme; LDH: lactate dehydrogenase; HBDH: Alpha Hydroxybutyrate Dehydrogenase; K+: Potassium lon Concentration; Na+: Sodium Ion Concentration; CRP:C-Reactive Protein; PCT: Procalcitonin; ESR: Erythrocyte Sedimentation Rate; P<0.05 is considered statistically significant, $P>0.05$ means the difference is not statistically significant.

The ratio of male to female children was 1.74:1 (191:110). There was no significant difference in age, weight, sex and place of residence, the highest body temperature-Tmax $\left({ }^{\circ} \mathrm{C}\right)$ during fever and the time from onset to use of IVIG-time(d) between the IVIG insensitive group and the IVIG sensitive group. The levels of N\%, B\%, ALT, AST and PCT in the IVIG insensitive group were significantly higher than those in the IVIG sensitive group, and the levels of L\%, M\%, HCT, MCH, CK and $\mathrm{Na}+$ were significantly lower than those in the IVIG sensitive group $(\mathrm{P}<0.05)$.There was no significant difference in the levels of WBC, E\%, RBC, HGB, MCV, MCHC, PLT, CK-MB, LDH, HBDH, K+, CRP and ESR between the two $\operatorname{groups}(\mathrm{P}>0.05)$ (Table 1$)$

\section{Binary Logistic Regression Analysis To Determine Risk Factors For IVIG Resistance}

Whether IVIG resistance occurs was regarded as a dependent variable and all the laboratory indicators were introduced as independent variables into binary logistic regression analysis. The results showed that the regression coefficients of AST and PCT were statistically significant and the relative risk of them were 1.011 and 1.128, respectively. AST and PCT were independent risk factors for IVIG resistance in children with KD (Table 2)
Table 2: Binary logistic regression analysis of risk factors for IVIG unresponsive KD

\begin{tabular}{|c|c|c|c|c|}
\hline & $\beta$ value & P value & OR value & 95\%CI of OR value \\
\hline constant & -4.152 & 0 & 0.016 & \\
\hline AST & 0.011 & 0.001 & 1.011 & $1.004-1.017$ \\
\hline PCT & 0.12 & 0.002 & 1.128 & $1.046-1.215$ \\
\hline
\end{tabular}

Note: $\beta$ : Regression Coefficient; OR: Relative Risk; Cl: Confidence Interval; AST: Aspartate Aminotransferase; PCT: Procalcitonin; $\mathrm{P}<0.05$ means statistically significant.

\section{AST and PCT Predict IVIG Resistance}

The ROC curve was used to determine the optimal cutoff value of AST and PCT for predicting the occurrence of IVIG resistance in $\mathrm{KD}$. The area under the curve of AST is 0.789 ; when AST $\geq 29.5 \mathrm{U} / \mathrm{L}$, the efficacy of predicting IVIG resistance is predicted to be the highest, and the sensitivity and specificity are $91.7 \%$ and $64.4 \%$, respectively. The area under the curve of PCT is 0.786 ; when $\mathrm{PCT} \geq 1.70 \mathrm{ng} / \mathrm{ml}$, the prediction efficiency is the highest, and the sensitivity and specificity are $91.7 \%$ and $62.3 \%$, respectively. (Figures 1 \& 2). 


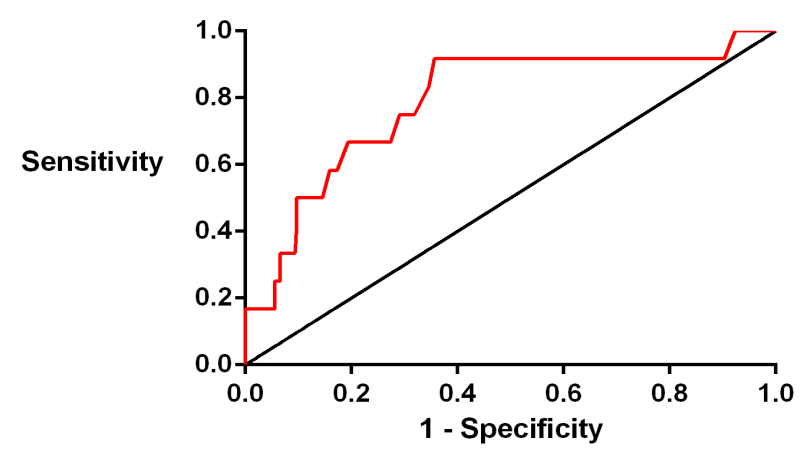

Figure 1: ROC curve of AST predicting IVIG Resistance.

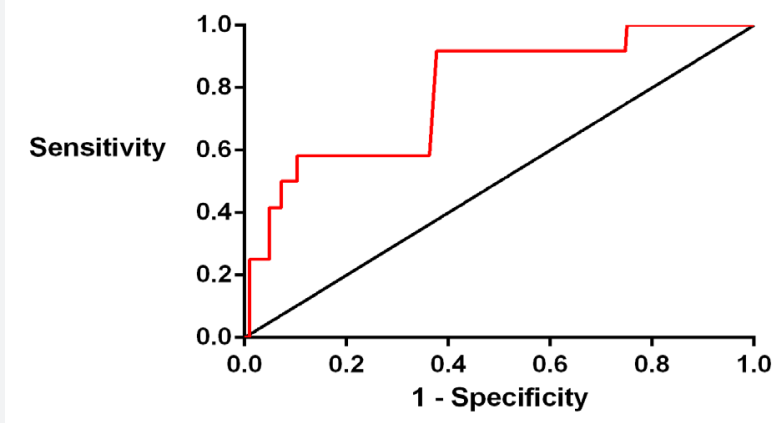

Figure 2: ROC curve of PCT predicting IVIG Resistance.

\section{Discussion}

Kawasaki disease(KD) is a systemic vasculitis in young children which is still not being diagnosed as frequently as it should be in developing countries [5]. About $20 \%-25 \%$ of untreated Kawasaki disease patients develop coronary artery complications and the prognosis of Kawasaki disease is closely related to coronary artery damages including and their severity. And a statement issued in 2017 by the American Heart Association on the diagnosis, treatment and long-term management of Kawasaki disease points out that coronary artery dilatation (CAL) with a $\mathrm{Z}$ value (that is, the diameter of the coronary artery corrected by body surface area) less than 2.5 is the most common, with an incidence of $32 \%$ to $50 \%$ which is higher than previously thought [6].Though some centers in India (including Chandigarh, Kolkata and Mumbai) have started using $\mathrm{Z}$ scores for assessment of coronary artery diameters, this is still not a routine practice in many parts of the country and other developing countries [7]. Now IVIG combined with oral aspirin therapy has become a recognized standard therapy, but some children with KD do not respond to the first dose of IVIG treatment and need the second dose of IVIG or hormone therapy. Recent studies have shown that the incidence of IVIG nonresponsive $\mathrm{KD}$ is increasing year by year [8] and non-response to IVIG is a risk factor for developing coronary artery disease [9]. It is important to identify this subset of patients because their chances of coronary artery abnormalities (CAA) are high [1]. Therefore, it is of great significance to find the predictive factors of IVIG nonresponse for children with high-risk KD to get timely and accurate treatment, to prevent the occurrence of serious complications and to reduce medical and family burden.

There are many studies on the predictive factors of IVIG resistance, but there is no consistent conclusion. Domestic related studies have found that WBC, N\%, CRP, ESR, ALB, HGB, TBil, Na+, NT-proBNP, age $<6$ months, rash and extremity edema are independent risk factors for IVIG resistance in children with KD[10-12]. Japanese scholars Kobayashi et al. [13], Sano et al. [14] and Egami et al. [15] respectively created a common scoring model for predicting IVIG resistance. A meta-analysis made by Baek et al. [16] showed that higher TBil, PMN, brain natriuretic peptide(BNP), AST, ALT, CRP, lower Na+ and ALB can predict IVIG non-responsive Kawasaki disease, while WBC,PLT and ESR have no predictive performance. The latest meta-analysis [17] showed that in addition to the effective predictors found by Baek et al. [16], the use of IVIG within 4 days after onset of KD, increased ESR, decreased HGB and PLT, oral mucosal changes, cervical lymphadenopathy, swelling of the extremities and polymorphic rash were also risk factors for IVIG resistance.

The subjects included in the above studies are different in race, region and epidemiology such as infection, and the laboratory indicators included in some studies are relatively single; in addition, there are some limitations in the existing predictive indicators, such as NT-proBNP, PMN, etc. cannot be routinely tested, the level of $\mathrm{Na}+$ may be affected by fever, irritability and poor eating in the acute stage of KD, non-specific inflammatory 
indicators WBC,CRP which have poor specificity are significantly elevated in KD, bacterial infections and autoimmune diseases [18]. In addition to the comprehensive statistical analysis of IVIG resistance-related routine test indexes, the study included PCT to obtain more valuable findings so as to early identify IVIG resistance high-risk children in the region and carry out early prevention and intervention.

This study shows that AST and PCT are independent risk factors for IVIG resistance in children with KD. When AST $\geq$ $29.5 \mathrm{U} / \mathrm{L}$, the sensitivity and specificity for predicting IVIG resistance are the highest; when PCT $\geq 1.70 \mathrm{ng} / \mathrm{ml}$, the sensitivity and specificity for predicting IVIG resistance are the highest. AST is mainly distributed in the myocardium, followed by liver, skeletal muscle and kidney tissue. Subclinical liver damage is common in patients with $\mathrm{KD}$, but its mechanism is unclear. The possible factors include systemic inflammation, vasculitis, toxin effects of anti-inflammatory drugs or the combination of above factors [19]. Abnormal liver function may reflect the severity of persistent KD inflammation to a certain extent and thus is related to the therapeutic effect of IVIG [20]. The results of Chantasiriwan et al. [21] in Thailand and Kim et al. [22] in Korea are similar to our conclusion, that is, the elevated AST level is related to the occurrence of IVIG resistance in children with KD. A meta-analysis by Liu et al. [20] found that levels of serum TBil, ALT, AST, and GGT were significantly higher in the IVIG non-responsive group of KD children than that in the IVIG-responsive group and liver function abnormalities were significantly associated with IVIG resistance in KD. There is no conclusion on the mechanism of high level of AST in IVIG non-responders. It is speculated that the persistent inflammatory reaction of KD children with IVIG resistance is more intense. Under the influence of more inflammatory factors and cellular mediators, the enhancement of the permeability of hepatic sinusoidal endothelial cells leads to a significant increase in the level of AST.

Our study is consistent with the results of Dominguez et al. [23], which suggests that PCT can help screen children with high risk of IVIG resistance. Procalcitonin PCT is a propeptide of calcitonin and an important inflammatory response marker in acute stage with specificity higher than CRP and ESR.PCT elevation is seen in bacterial or non-bacterial infections: PCT can be secreted by neuroendocrine cells such as $\mathrm{C}$ cells of thyroid and pancreatic tissue after bacterial infection and changes with the progression of infection; PCT can also rise in Kawasaki disease, acute viral diarrhea, early neonatal, multiple injuries and postoperative. The first study to explore the prediction of KD by PCT [18] showed that PCT levels in patients with KD and bacterial infections were significantly higher than those in patients with autoimmune diseases or virus infections, and PCT could be used to predict coronary aneurysms. It has been proved that PCT can inhibit the release of tumor necrosis factor- $\alpha$ (TNF- $\alpha$ ) and elevated PCT may play a certain role in alleviating vasculitis caused by elevated TNF- $\alpha$ in KD patients [18], furthermore, the level of TNF- $\alpha$ is significantly higher in IVIG non-responders and TNF- $\alpha$ can predict IVIG resistance [24]. As a result, elevated PCT levels in children with IVIG non-responsive KD may be associated with more severe and longer duration systemic vasculitis caused by more TNF- $\alpha$ release. However, the specific mechanism needs further study.

In summary, our study conducted a comprehensive statistical analysis of laboratory routine test ind exes related to IVIG resistance and included the PCT index but did not conduct a prospective study. In addition, incomplete clinical data in some cases may cause bias in statistical analysis. Therefore, future multicenter prospective cohort studies on IVIG resistant KD predictors are more persuasive. We still need to consider how to effectively prevent the occurrence of IVIG nonresponse after screening high risk KD children with IVIG resistance by using predictive indexes, whether to continue to apply the original standard treatment plan for these children and so on.

\section{References}

1. Singh S, Sharma A, Jiao FY (2016) Kawasaki Disease: Issues in Diagnosis and Treatment--A Developing Country Perspective. Indian J Pediatr 83(2): 140-145.

2. Agarwal S, Agrawal Dk (2017) Kawasaki Disease: Etiopathogenesis and Novel Treatment Strategies. Expert Rev Clin Immunol 13(3): 247258.

3. Lee SM, Lee JB, GO YB Song HY, Lee BJ, et al. (2014) Prediction of Resistance to Standard Intravenous Immunoglobulin Therapy in Kawasaki Disease. Korean Circ J 44(6): 415-422.

4. Newburger JW, Takahashi M, Gerber MA, Gewitz MH, Tani LY, et al. (2004) Diagnosis, treatment, and long-term management of Kawasaki disease: a statement for health professionals from the Committee on Rheumatic fever, Endocarditis and Kawasaki Disease, Council on Cardiovascular Disease in the Young, American Heart Association. Circulation 110: 2747-2771.

5. Jiao FY, Yan XH, Wu XF (2016) The Xi'an Experience of Kawasaki Disease-Lessons Learnt over 5 years. Indian J Pediatr 83(10): 11951196.

6. MC Crindle BW, Rowley AH, Newburger JW, Burns JC, Bolger AF, et al. (2017) Diagnosis, treatment, and long-term management of Kawasaki disease: a scientific statement for health professionals from the American Heart Association. Circulation 135(17): e927-e999.

7. Jiao FY, Jindal AK, Pandiarajan V, Raju Khubchandani, Nutan Kamath,et al. (2017) The emergence of Kawasaki disease in India and China. Glob Cardiol Sci Pract 2017(3).

8. Yoshimura K, Kimata T, Mine K, Uchiyama T, Tsuji S, et al. (2013) $\mathrm{N}$-terminal pro-brain natriuretic peptide and risk of coronary artery lesions and resistance to intravenous immunoglobulin in Kawasaki disease. J Pediatr 162(6): 1205-1209.

9. JCS Joint Working Group (2014) Guidelines for Diagnosis and Management of Cardiovascular Sequelae in Kawasaki Disease (JCS 2013). Digest version. Circ J 78(10): 2521-2562.

10. Yang S, Song RX, Zhang JM, Xiaohui Li, Caifeng Li (2018) Predictive tool for intravenous immunoglobulin resistance of Kawasaki disease in Beijing. Arch Dis Child.

11.Xie T, Wang Y, Fu S, Wang W, Xie C, et al. (2017) Predictors for intravenous immunoglobulin resistance and coronary artery lesions in Kawasaki disease. Pediatr Rheumatol Online J 15(1):17.

12. Tang YJ, Yan WH, Sun L, Huang J, Qian W, et al. (2006) Prediction of intravenous immunoglobulin resistance in Kawasaki disease in an East China population. Clin Rheumatol 35: 2771-2776. 
13. Kobayashi T, Inoue Y, Takeuchi K, Okada Y, Tamura K, et al. (2006) Prediction of intravenous immunoglobulin unresponsiveness in patients with Kawasaki disease. Circulation 113(22): 2606-2612.

14. Sano T, Kurotobi S, Matsuzaki K, Yamamoto T, Maki I, et al. (2007) Prediction of non-responsiveness to standard high-dose gammaglobulin therapy in patients with acute Kawasaki disease before starting initial treatment. Eur J Pediatr 166(2): 131-137.

15. Egami K, Muta H, Ishii M, Suda K, Sugahara Y, et al. (2006) Prediction of resistance to intravenous immunoglobulin treatment in patients with Kawasaki disease. J Pediatr 149(2): 237-240.

16. Baek JY, Song MS. (2016) Meta-analysis of factors predicting resistance to intravenous immunoglobulin treatment in patients with Kawasaki disease. Korean J Pediatr 59(2): 80-90.

17. Li X, Chen Y, Tang Y, Ding Y, Xu Q, et al. (2018) Predictors of intravenous immunoglobulin-resistant Kawasaki disease in children: a metaanalysis of 4442 cases. Eur J Pediatr 177(8): 1279-1292.

18. Okada Y, Minakami H, Tomomasa T, Kato M, Inoue Y, et al. (2004) Serum procalcitonin concentration in patients with Kawasaki disease. J Infect 48(2): 199-205.

19. Eladawy M, Dominguez SR, Anderson MS, Glodé MP. (2011) Abnormal liver panel in acute kawasaki disease. Pediatr Infect Dis J 30(2): 141144.

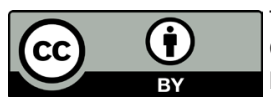

This work is licensed under Creative

Commons Attribution 4.0 License

DOI: 10.19080/NAPDD.2018.04.555637
20. Liu L, Yin W, Wang RG, Sun D, He X et al. (2016) The prognostic role of abnormal liver function in IVIG unresponsiveness in Kawasaki disease: a meta-analysis. Inflamm Res 65(2): 161-168.

21. Chantasiriwan N, Silvilairat S, Makonkawkeyoon K, Pongprot Y, Sittiwangkul R (2018) Predictors of intravenous immunoglobulin resistance and coronary artery aneurysm in patients with Kawasaki disease. Paediatr Int Child Health 38(3): 209-212

22. Kim MK, Song MS, Kim GB (2018) Factors Predicting Resistance to Intravenous Immunoglobulin Treatment and Coronary Artery Lesion in Patients with Kawasaki Disease: Analysis of the Korean Nationwide Multicenter Survey from 2012 to 2014. Korean Circ J 48(1): 71-79.

23. Dominguez SR, Martin B, Heizer H, Jone PN, Tong S, et al. (2016) Procalcitonin (PCT) and Kawasaki Disease: Does PCT Correlate With IVIG-Resistant Disease, Admission to the Intensive Care Unit, or Development of Coronary Artery Lesions? J Pediatric Infect Dis Soc 5(3): 297-302.

24. Hu P, Jiang GM, Wu Y, Huang BY, Liu SY, et al. (2017) TNF- $\alpha$ is superior to conventional inflammatory mediators in forecasting IVIG nonresponse and coronary arteritis in Chinese children with Kawasaki disease .Clin Chim Acta 471: 76-80.

\section{Your next submission with Juniper Publishers will reach you the below assets}

- Quality Editorial service

- Swift Peer Review

- Reprints availability

- E-prints Service

- Manuscript Podcast for convenient understanding

- Global attainment for your research

- Manuscript accessibility in different formats

( Pdf, E-pub, Full Text, Audio)

- Unceasing customer service

Track the below URL for one-step submission https://juniperpublishers.com/online-submission.php 